\title{
Thoracic Exploration for Mediastinal Parathyroids requires a Multidisciplinary Approach
}

Jamie C Mitchell, Mira Milas, Eren Berber, Allan Siperstein, Joyce Shin, Thomas Rice, Tomislav Mihaljevic, David Mason

\begin{abstract}
Mediastinal parathyroid disease requiring a thoracic surgical approach is a rare and challenging clinical entity. The objective of this study was to review our experience treating these patients, highlighting the importance of a multidisciplinary approach. Seven patients required a thoracic approach for mediastinal parathyroid disease between 1999 and 2010. All patients achieved biochemical cure with varying combinations of preoperative and intraoperative localizing studies interpreted by a multidisciplinary team, including radiologists, thoracic and endocrine surgeons, being required in each patient.
\end{abstract}

Keywords: Mediastinum, Parathyroid imaging, Neuroendocrine tumor, Surgical exposure.

How to cite this article: Mitchell JC, Milas M, Berber E, Siperstein A, Shin J, Rice T, Mihaljevic T, Mason D. Thoracic Exploration for Mediastinal Parathyroids requires a Multidisciplinary Approach. World J Endoc Surg 2012;4(3): 87-92.

\section{Source of support: Nil}

\section{Conflict of interest: None}

\section{INTRODUCTION}

Primary hyperparathyroidism (PHPT) results from hyperactivity of one or more parathyroid glands, with a prevalence of between one and four in 1,000 people. While PHPT can occur at any age, it is more common in patients above age 50 and postmenopausal women. ${ }^{1}$ The majority of parathyroid glands are located in the cervical region, however, they can be located in ectopic locations in up to $25 \%$ of cases, including the mediastinum. ${ }^{2}$ The majority of mediastinal parathyroid glands can be excised via a cervical incision, with a minority requiring a thoracic approach.

Patients requiring thoracic approaches for excision of ectopic parathyroid glands represent a unique challenge with respect to preoperative and intraoperative localization of the ectopic mediastinal gland. The majority of these patients have already undergone one or more unsuccessful cervical or mediastinal explorations, adding to their clinical complexity. In many instances, multiple preoperative localization studies are required to confidently identify the ectopic gland. Additionally, despite confident preoperative localization, intraoperative localization can also be challenging due to the reoperative nature of many of these cases, and the visual subtlety of mediastinal parathyroid gland tissue.
Due to the rarity of this clinical situation as well as its multiple levels of complexity, evaluation by a number of specialists is often required for successful treatment of these patients. The purpose of this study was to review our experience treating patients requiring a transthoracic approach to treat ectopic mediastinal parathyroid glands causing PHPT, focusing on the importance of a multidisciplinary approach to management.

\section{MATERIALS AND METHODS}

The study was conducted by retrospective review of a prospectively maintained database of all patients undergoing surgery for primary hyperparathyroidism between 1999 and 2010 to identify all patients who required a transthoracic approach. Preoperative evaluation of patients prior to surgery at our institution followed a clinical algorithm summarized in Figure 1. A number of preoperative parameters were reviewed, including previous surgical history relating to parathyroid disease and all preoperative localizing studies obtained prior to the thoracic exploration performed at our institution. Preoperative localizing studies obtained at our institution included sestamibi-iodine subtraction scans with single photon emission computed tomography (SPECT) imaging and computed tomography (CT) colocalization utilizing technetium-99 sestamibi and I-123 as radiopharmaceuticals (MIBI), contrast-enhanced magnetic resonance imaging (MRI) of the neck and chest, contrast-enhanced CT scan of the neck and chest, selective venous sampling for parathyroid hormone from the cervical and mediastinal veins. All patients underwent sestamibiiodine subtraction scans, with additional tests being

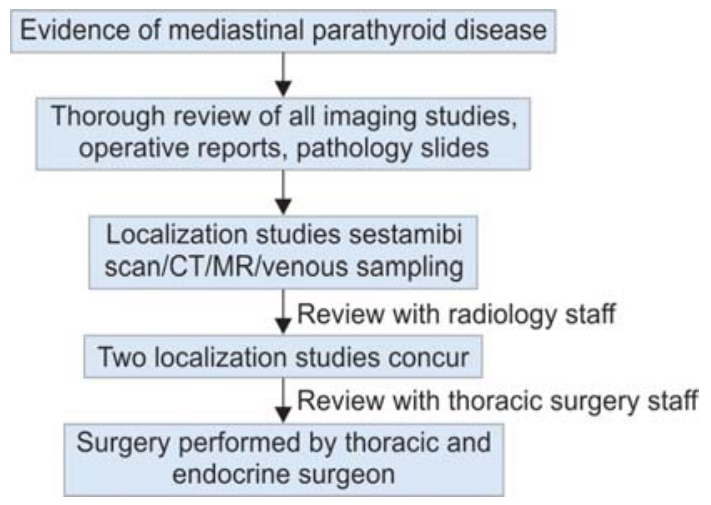

Fig. 1: Clinical algorithm 
performed based on the clinical scenario. Methods of intraoperative localization of diseased mediastinal parathyroid glands were reviewed as well, including number of glands identified visually, identification by the use of a radioguided probe after preoperative sestamibi injection, identification by frozen section analysis and identification by permanent section analysis. All patients had intraoperative intact parathyroid hormone assayed before and after excision of mediastinal tissue. Postoperative outcome was also analyzed, including length of hospital stay, calcium, parathyroid hormone and vitamin D levels and perioperative complications.

Due to the limited sample size of seven patients, statistical analyses were not performed.

\section{RESULTS}

During the study period 1,600 patients underwent surgery for PHPT at our institution, with seven requiring transthoracic exploration (0.004\%). Patients included four women and three men (57 and 43\% respectively), with ages ranging from 25 to 82 (mean, 52). All seven patients had undergone at least one previous parathyroid exploration, with three patients (43\%) having undergone multiple failed parathyroid surgeries (1-4, mean 1.9). Previous surgical history related to parathyroid disease including operative findings is summarized in Table 1.

\section{Preoperative Localization}

Three patients had ectopic glands located in the anterior mediastinum (43\%), three patients had glands located in the middle mediastinum (43\%), and one patient had a gland located in the posterior mediastinum (14\%). Table 2 summarize the specific locations of mediastinal glands in all seven patients and the surgical approach to the mediastinum. A total of 18 preoperative localization studies were performed on seven patients. The number of studies obtained per patient ranged from one to four with a mean of 2.4. Imaging studies were considered positive if clear visual evidence of an ectopic parathyroid gland was present after thorough review by the surgeon and radiologist. Venous sampling studies were considered positive if there was a two-fold increase in parathyroid hormone levels in one venous drainage basin compared with the others tested. Veins sampled during this study include superior, middle and inferior thyroid veins, high, middle and inferior jugular vein, subclavian veins, innominate veins and the superior vena cava. Tables 3 and 4 summarize the results of localizing studies. Examples of positive localizing studies are shown in Figures 2A to 3B.

\section{Intraoperative Localization}

Surgical approach included three upper hemisternotomies, two full median sternotomies, one right thoracoscopy, and

\begin{tabular}{cll} 
& \multicolumn{2}{c}{ Table 1: Summary of surgical history } \\
\hline Patient & Previous surgeries & Operative findings \\
\hline 1 & Bilateral neck exploration & RL excised, normal RU/LL, LU not identified \\
2 & Bilateral neck exploration & LU/LL/RU/RL normal \\
3 & Bilateral neck exploration & LU/LL/RU normal, RL not identified \\
& Bilateral neck exploration & No gland identified \\
4 & Bilateral neck exploration & LU/RU/RL normal, LL not identified \\
& Left thoracotomy & No gland identified \\
& Median sternotomy & No gland identified \\
5 & Left thoracotomy & No gland Identified \\
6 & Bilateral neck exploration & LU/LL/RU/RL normal \\
7 & Right neck exploration & RU/RL normal \\
& Bilateral neck exploration & Normal LU excised, LL/RU/RL not identified \\
& Bilateral neck exploration & No gland identified \\
\hline
\end{tabular}

LL: Left lower parathyroid gland; LU: Left upper parathyroid gland; RL: Right lower parathyroid gland; RU: Right upper parathyroid gland

Table 2: Location of ectopic parathyroid glands

\begin{tabular}{cll}
\multicolumn{2}{c}{ Table 2: Location of ectopic parathyroid glands } \\
\hline Patient & Gland location & Surgical approach \\
\hline 1 & Lateral to aortic root & Upper hemisternotomy \\
2 & Lateral to innominate vein/SVC junction & Upper hemisternotomy \\
3 & Inferior to aortic arch at carina & Upper hemisternotomy \\
4 & Aortopulmonary window & Median sternotomy \\
5 & Aortopulmonary window & Median sternotomy \\
6 & Anterior pericardium & Robot-assisted left thoracoscopy \\
7 & Lateral to esophagus at level of azygos vein & Right thoracoscopy \\
\hline
\end{tabular}

SVC: Superior vena cava 


\begin{tabular}{cll}
\hline & & \multicolumn{1}{c}{ Table 3: Summary of localizing studies } \\
\hline Patient & Localizing study & \multicolumn{1}{c}{ Results } \\
\hline 1 & Sestamibi-iodine subtraction scan & Signal anterior to aortic root at T-6 \\
2 & Sestamibi-iodine subtraction scan & Negative study \\
& Contrast-enhanced CT scan & Enhancing mass adjacent to innominate/SVC junction \\
3 & Venous sampling & PTH in SVC 3-fold higher than cervical veins \\
& Sestamibi-iodine subtraction scan & Signal on right inferior to aortic arch at level of carina \\
& MRI & Negative study \\
4 & Contrast-enhanced CT scan & Enhancing mass right anterior mediastinum \\
& Sestamibi-iodine subtraction scan \#1 & Negative study \\
& Venous sampling & Negative study \\
& Sestamibi-iodine subtraction scan \#2 & Signal in aortopulmonary window \\
& Sestamibi-iodine subtraction scan & Signal in aortopulmonary window \\
& Venous sampling & Negative study \\
& Sestamibi-iodine subtraction scan & Signal in anterior pericardium at RV outflow tract \\
& MRI & Signal in anterior pericardium at RV outflow tract \\
& Venous sampling & Negative study \\
& Sestamibi-iodine subtraction scan & Signal in posterior mediastinum at TE groove \\
& Contrast-enhanced CT scan & Enhancing mass in TE groove \\
& MRI & Enhancing mass in TE groove
\end{tabular}

PTH: Parathyroid hormone; RV: Right ventricular; SVC: Superior vena cava; TE: Tracheoesophageal

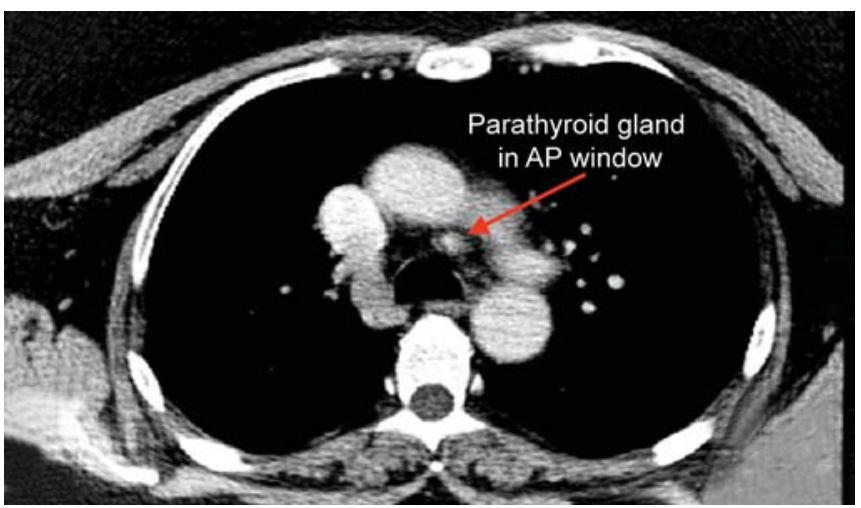

Fig. 2A: Contrast-enhanced CT scan

one robot-assisted left thoracoscopy. Five patients underwent preoperative sestamibi injection preoperatively for the purposes of radioguided localization of the ectopic parathyroid gland intraoperatively. Of these patients, radioguided probe was not useful in localizing the parathyroid gland in situ. In two of five patients, it was helpful in confirming parathyroid tissue in excised tissue. Parathyroid glands were only identified visually during mediastinal exploration in two of seven patients (29\%). The number of specimens removed ranged from one to 11 (mean, 4.4). Hypercellular parathyroid tissue was identified on frozen section analysis of excised tissue in six of seven patients (86\%), while in one patient the only indication of successful excision of the ectopic gland during surgery was a drop in intraoperative parathyroid hormone levels. Hypercellular parathyroid tissue was confirmed on permanent section of excised tissue in all patients. Table 5 summarizes intraoperative localizing studies.

\section{Outcomes}

All seven patients had normalization of their calcium and parathyroid hormone levels on postoperative day 1. Calcium

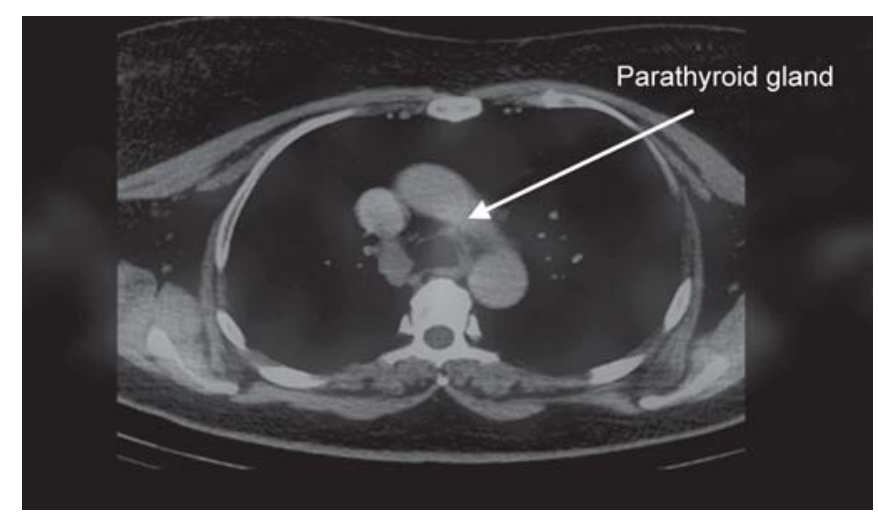

Fig. 2B: CT/sestamibi fusion images

and parathyroid hormone levels remained normalized in all patients during follow-up, which ranged from 2 weeks to 3 years. Hospital stay ranged between 1 and 5 days with a mean of 2.7 days. Mean hospital stay for open procedures was 3.2 days compared with 1.5 days for thoracoscopic procedures. Among open procedures, mean hospital stay for hemisternotomies was 2.3 days compared with 4.5 days for full sternotomies. Two patients had recurrent laryngeal nerve palsies after mediastinal exploration at our institution (28\%). In both of these patients the ectopic gland location was in the AP window. Another patient had a recurrent laryngeal nerve palsy from one of their prior parathyroid operations, thus $43 \%$ of the study population suffered a recurrent laryngeal nerve injury prior to achieving biochemical cure. Two patients had transient hypocalcemia in the perioperative period while receiving intravenous fluids with no patients having prolonged hypocalcemia.

\section{DISCUSSION}

Mediastinal parathyroid disease requiring a thoracic exploration is a rare clinical entity, but one that can involve a great deal of complexity. The majority of these patients 
Table 4: Sensitivity of preoperative localizing studies

\begin{tabular}{lccc}
\hline Imaging study & N & Positive study & Sensitivity \\
\hline CT scan & 6 & 5 & $83 \%$ \\
MRI & 3 & 2 & $67 \%$ \\
Sestamibi-iodine & 8 & 6 & $71 \%$ \\
subtraction scan & & & \\
Venous sampling & 4 & 1 & $25 \%$ \\
\hline
\end{tabular}

CT: Computed tomography; MRI: Magnetic resonance imaging

have negative localizing studies during their initial evaluations, which lead to failed cervical and/or thoracic explorations, and consequently a reoperative situation at the time of definitive treatment. Additionally, due to the reoperative nature of many of these cases and the visual subtlety of some of these glands, intraoperative identification of ectopic parathyroid tissue in the mediastinum during thoracic exploration can be challenging even in the setting of clearly localizing imaging studies. When faced with these difficult patients, therefore, it is important and often necessary to utilize all available tools to achieve clinical cure.

Due to the complexity of mediastinal parathyroid disease, it becomes more important to utilize a combination of both structural (US, CT) and functional (MIBI) localization studies in order to confidently identify the diseased gland prior to embarking on a mediastinal exploration. ${ }^{3-6}$ The potential morbidity related to a thoracic exploration can be significant, and as our small case series demonstrates, intraoperative identification of these ectopic glands can be very challenging even in the setting of clear localizing studies. Consequently, clear confirmation of the mediastinal gland location by a minimum of two localizing studies, including at least one functional study, is recommended. ${ }^{7-10}$

While Table 4 summarizes the overall sensitivity of the various localizing studies used in our seven patients, the purpose of this study was not to identify the 'best' imaging study for this patient population. Each patient is unique, and each required a different combination of studies to provide the confidence required to proceed with mediastinal exploration. Perhaps more important was the multidisciplinary evaluation of these localizing studies by radiologists, thoracic and endocrine surgeons to determine when it was appropriate to proceed with surgery or whether additional testing was required, as well as the optimal surgical approach. ${ }^{11-13}$

A number of tools are available to aid in the confirmation that the abnormal parathyroid tissue has been excised, including the use of the radioguided probe in conjunction with preoperative sestamibi injection, ${ }^{14,15}$ frozen section analysis of excised tissue and intraoperative PTH assays. The difficulty in visually identifying the mediastinal parathyroid gland in many of these cases was somewhat surprising, particularly given the participation of an experienced endocrine surgeon in each case. The

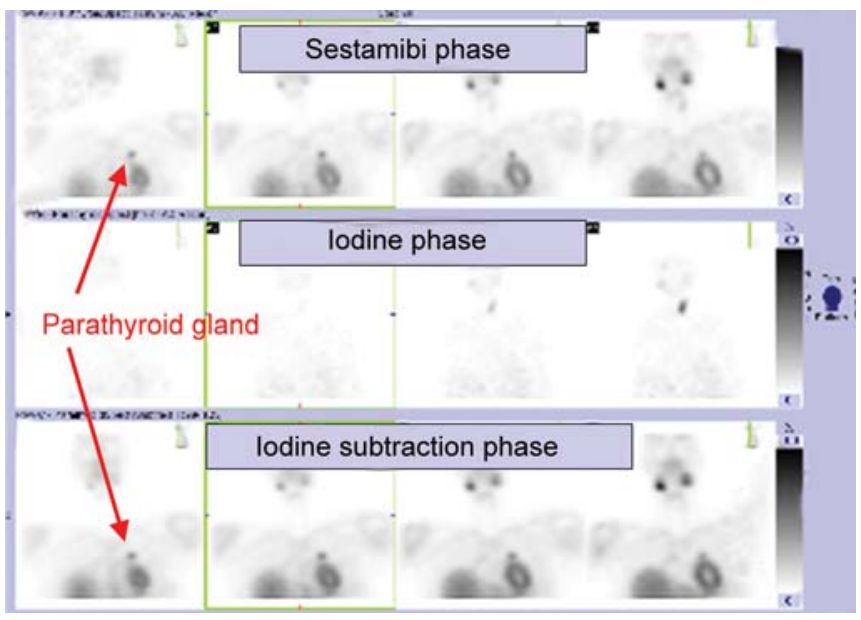

Fig. 3A: Sestamibi-iodine subtraction scan

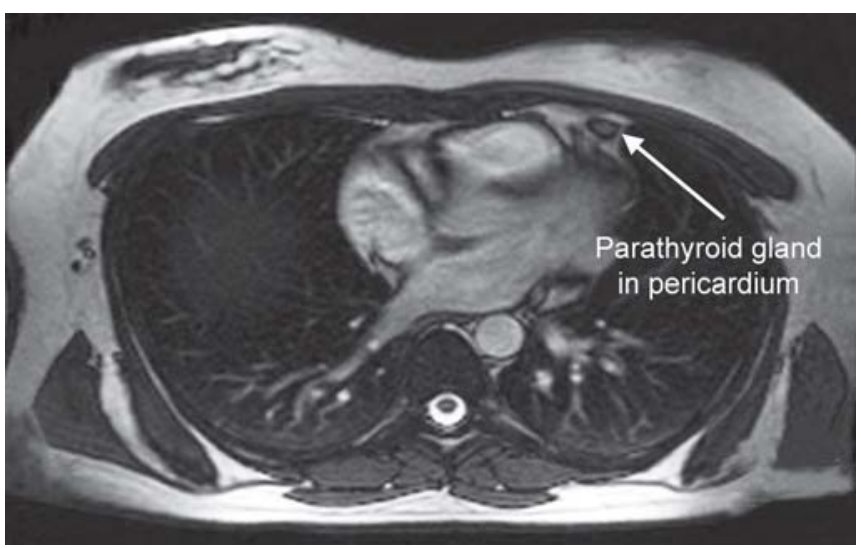

Fig. 3B: Magnetic resonance imaging

\begin{tabular}{ccccccc}
\multicolumn{1}{c}{ Table 5: Summary of intraoperative localization } \\
\hline Patient & Inspection & $\begin{array}{c}\text { Radioguided } \\
\text { probe in situ }\end{array}$ & $\begin{array}{c}\text { Radioguided } \\
\text { probe ex vivo }\end{array}$ & $\begin{array}{c}\text { Number of } \\
\text { specimens }\end{array}$ & $\begin{array}{c}\text { Frozen section } \\
\text { Permanent } \\
\text { section }\end{array}$ \\
\hline 1 & No & No & No & 11 & Yes & Yes \\
2 & No & No & No & 4 & Yes & Yes \\
3 & Yes & N/A & N/A & 1 & Yes & Yes \\
4 & No & No & Yes & 5 & No & Yes \\
5 & No & No & No & 7 & Yes & Yes \\
6 & No & No & Yes & Yes & Yes \\
7 & Yes & N/A & N/A & 1 & Yes & $7 / 7(100 \%)$ \\
\hline
\end{tabular}


reoperative nature of some of these cases with extensive scarring and perhaps the relatively large degree of mediastinal fibrofatty lymphatic tissue were likely contributing factors, however, the reason for this difficulty is not entirely clear.

Of the five patients where the radioguided probe was utilized intraoperatively, four had uptake on the preopertive sestamibi scan. Use of the radioguided probe was of limited utility in our series, assisting in the identification of parathyroid tissue in only two cases, and then only in excised tissue. The inability of the probe to consistently identify the offending parathyroid tissue in situ in this series may be due to the relatively higher background activity in the mediastinum due to uptake of radiopharmaceutical in the cardiac structures compared with the cervical space. Despite its limitations in this series, use of the gamma probe was helpful in two of the cases. Chen et al showed that radioguided techiques were equally effective whether or not the preoperative sestamibi scan showed uptake, ${ }^{14}$ arguing for its routine use irrespective of the results of preopertive sestamibi scanning to possibly aid in intraoperative localization in these difficult cases.

The two patients with parathyroid lesions in the AP window exhibited signs of recurrent laryngeal nerve palsy postoperatively, although it is not felt that this was due to the gland location per se. In one of these cases, ours was the fourth mediastinal exploration the patient had undergone, and the extensive scarring precluded visualization of the nerve anatomy during the dissection. While no nerve injury was recognized intraopertively, the patient had a left vocal cord paresis postoperatively requiring medialization. In the other case, while the nerve was identified, extensive dissection was carried out as the parathyroid tissue could not be visually identified. Much of the fibrofatty tissue along the nerve's path had to be excised, and the postoperative nerve palsy was felt to likely be temporary in nature.

\section{SUMMARY}

The seven cases presented here demonstrate the potential challenges of treating mediastinal parathyroid disease. Successful management of these complex pateints requires a coordinated, multidisciplinary approach to management, where endocrine surgeons, thoracic surgeons, pathologists and radiologists work in concert utilizing all available tools for preoperative and intraoperative localization of the diseased gland to maximize the chance for clinical cure.

\section{REFERENCES}

1. Khan AA, Bilezikian JP, Potts JT. Third international workshop on asymptomatic primary hyperparathyroidism. The diagnosis and management of asymptomatic primary hyperparathyroidism revisited. J Clin Endocrinol Metab 2009;94(2):333-34.
2. Iacabone M, Mondi I, Viel G, et al. The results of surgery for mediastinal parathyroid tumors: A comparative study of 63 patients. Langenbecks Arch Surg 2010;395:947-53.

3. van Heerden JA, Grant CS. Surgical treatment of primary hyperparathyroidism: An institutional perspective. World J Surg 1991;15:688-92.

4. Allendorf J, DiGorgi M, Spanknebel K, et al. 1112 consecutive bilateral neck explorations for primary hyperparathyroidism. World J Surg 2007;31:2075-80.

5. Chen H, Sokoll LJ, Udelsman R. Outpatient minimally invasive parathyroidectomy: A combination of sestamibi-SPECT localization, cervical block anesthesia, and intraoperative parathyroid hormone assay. Surgery 1999;126:1016-21.

6. Arici C, Cheah WK, Ituarte $\mathrm{PH}$, et al. Can localization studies be used to direct focused parathyroidect operations? Surgery 2001;129:720-29.

7. Shah S, Win Z, Al-Nahhas A. Multimodality imaging of the parathyroid glands in primary hyperparathyroidism. Minerva Endocrinol 2008;33(3):193-202.

8. Sukan A, Reyhan M, Aydin M, et al. Preoperative evaluation of hyperparathyroidism: The role of dual-phase parathyroid scintigraphy and ultrasound imaging. Ann Nucl Med 2008;22: 123-31.

9. Siperstein A, Berber E, Mackey R, et al. Prospective evaluation of sestamibi scan, ultrasonography, and rapid PTH to predict the success of limited exploration for sporadic primary hyperparathyroidism. Surgery 2004;136:872-80.

10. Solorzano CC, Carneiro-Pla DM, Irvin GL 3rd. Surgeonperformed ultrasonography as the initial and only localizing study in sporadic primary hyperparathyroidism. J Am Coll Surg 2006;202:18-24.

11. Liu RC, Hill ME, Ryan Jr JA. One-gland exploration for mediastinal parathyroid adenomas: Cervical and thoracoscopic approaches. Am J Surg 2005;189:601-05.

12. Randone B, Costi R, Scatton O, et al. Thoracoscopic removal of mediastinal parathyroid glands: A critical appraisal of an emerging technique. Ann Surg 2010;251:717-21.

13. Ismail M, Maza S Sweirzy $M$, et al. Resection of ectopic mediastinal parathyroid glands with the da Vinci robotic system. Br J Surg 2010;97:337-43.

14. Chen H, Sippel R, Schaefer S. The effectiveness of radioguided paratyhroidectomy in patients with negative technetium Tc 99msestamibi scans. Arch Surg 2009;144(7):643-48.

15. Norman J, Chheda H. Minimally invasive parathyroidectomy facilitated by intraoperative nuclear mapping. Surgery 1997;122: 998-1003.

\section{ABOUT THE AUTHORS}

\section{Jamie C Mitchell (Corresponding Author)}

Assistant Professor of Surgery, Section of Endocrine Surgery Cleveland Clinic Lerner College of Medicine, 9500 Euclid Avenue F-20, Cleveland, Ohio-44195, USA, Phone: 216-445-9713 Fax: 216-636-0662, e-mail: mitchej5@ccf.org

\section{Mira Milas}

Professor of Surgery (Oregon Health and Sciences University), Section of Endocrine Surgery, Cleveland Clinic Lerner College of Medicine Cleveland, Ohio, USA 


\section{Eren Berber}

Associate Professor of Surgery, Section of Endocrine Surgery Cleveland Clinic Lerner College of Medicine, Cleveland, Ohio, USA

\section{Allan Siperstein}

Professor of Surgery, Section of Endocrine Surgery, Cleveland Clinic Lerner College of Medicine, Cleveland, Ohio, USA

\section{Joyce Shin}

Assistant Professor of Surgery, Section of Endocrine Surgery Cleveland Clinic Lerner College of Medicine, Cleveland, Ohio, USA

\section{Thomas Rice}

Professor of Surgery, Section of Cardiothoracic Surgery, Cleveland Clinic Lerner College of Medicine, Cleveland, Ohio, USA

\section{Tomislav Mihaljevic}

Professor of Surgery, Section of Cardiothoracic Surgery, Cleveland Clinic Lerner College of Medicine, Cleveland, Ohio, USA

\section{David Mason}

Staff Surgeon, Section of Cardiothoracic Surgery, Cleveland Clinic Lerner College of Medicine, Cleveland, Ohio, USA 